\title{
Liderazgo transformacional y equidad de género: el caso de estudiantes de posgrado
}

\section{Eduardo Raúl Díaz Gómez*}

Fecha de recibido: 4 de abril de 2019

Fecha de aprobado: 16 de enero de 2020

Para citar: Díaz Gómez, E. R. (2020). Liderazgo transformacional y equidad de género: el caso de estudiantes de posgrado. Revista Universidad \& Empresa, 22(39), 1-21. https://doi.org/10.12804/revistas.urosario.edu.co/empresa/a.7854

\section{Resumen}

La representación de la mujer en roles de liderazgo incrementa en contextos organizacionales, educativos y políticos en México. Sin embargo, todavía existen barreras para la inclusión y equidad de género para mujeres que aspiran a desempeñarse como líderes. Estas parten de estereotipos que califican los estilos directivos de la mujer como comunales que, si bien fomentan el desarrollo de relaciones interpersonales, merman el cumplimiento de objetivos y el alto desempeño de equipos de trabajo que responden mejor a estilos gerenciales tradicionalmente relacionados con el género masculino. Existen estudios empíricos desarrollados con base en la teoría de liderazgo transformacional que desmienten la diferencia de actuaciones entre mujeres y hombres en puestos de liderazgo. La característica universal de la teoría transformacional de liderazgo la hace especialmente apropiada para comparar conductas entre grupos de género femenino y masculino, especialmente en estudios donde se adopta un diseño de auto-reporte. Este estudio utiliza un modelo de liderazgo transformacional para comparar la autopercepción de 309 estudiantes de posgrado entre mujeres y hombres. Los resultados sugieren que ambos grupos perciben su comportamiento de manera similar. Este resultado complementa otros estudios que sostienen que el género no influye sobre la conducta de líderes transformacionales.

Palabras clave: género; liderazgo; equidad; inclusión.

Profesor de tiempo completo en la Escuela de Administración y Negocios en CETYs Universidad. Es doctor en liderazgo por City University of Seattle con maestría en administración. Correo electrónico: eduardo.diaz@cetys.mx 


\title{
Transformational Leadership and Gender Equity: The Case of Graduate Students
}

\begin{abstract}
The role of women in leadership roles increases in organizational, educational, and political contexts in Mexico. However, barriers for aspiring female leaders and gender equity remain. These are the result of stereotypes that associate female leaders with communal behaviors that promote good interpersonal relationships but decrease goal attainment and performance within work teams. It is believed that these work teams typically respond better to traditional "male" leader behaviors. Empirical studies developed under transformational leadership theory refute the claim that female and male leaders behave differently. The universal characteristic of transformational leadership theory makes it appropriate for studies where female and male behaviors are compared, especially with self-report research designs. This study uses one transformational leadership model to compare the self-perceptions of 309 female and male graduate students. The results suggest that both groups perceive their behavior similarly. These results support the conclusions of other studies where gender was not found to moderate transformational leadership.
\end{abstract}

Keywords: gender; leadership; equity; inclusion.

\section{Liderança transformacional e equidade de gênero: o caso de estudantes de pós-graduação}

\section{Resumo}

A representação da mulher em posições de liderança incremente em contextos organizacionais, educativos e políticos no México. No entanto, ainda existem barreiras para a inclusão e equidade de gênero para mulheres que aspiram a se desempenhar como líderes. Estas barreiras partem de estereótipos que relacionam os estilos diretivos da mulher como comunais que, embora fomentam o desenvolvimento de relações interpessoais, diminuem o cumprimento de objetivos e o alto desempenho de equipes de trabalho que respondem melhor a estilos gerenciais tradicionalmente relacionados com o gênero masculino. Existem estudos empíricos desenvolvidos com base na teoria de liderança transformacional que desmentem que mulheres e homens atuam diferente em posições de liderança. A característica universal da teoria transformacional de liderança a faz especialmente apropriada para comparar condutas entre grupos de gênero feminino e masculino, especialmente em estudos onde se adota um desenho de auto-reporte. Este estuda utiliza um modelo de liderança transformacional para comparar a autopercepção de 309 estudantes de pós-graduação entre mulheres e homens. Os resultados sugerem que ambos os grupos percebem seu comportamento de maneira similar. Este resultado complementa outros estudos onde se sustenta que o gênero não influi sobre a conduta de líderes transformacionais.

Palavras-chave: gênero; liderança; equidade; inclusão.

\section{Introducción}

\author{
Las mujeres con estudios universitarios actualmente incrementan su participación en los \\ equipos gerenciales de organizaciones y en el escenario político en diferentes países,
}


pero su ascenso ha sido complejo y en muchos casos frustrante (Eagly \& Carli, 2012). Es razonable suponer que las mujeres sacrifican más que sus colegas hombres por obtener las mimas oportunidades de avanzar en los negocios y la política. A lo largo de este artículo se mencionarán algunos ejemplos de trabajos desarrollados por personal docente y administrativo en Instituciones de Educación Superior (IES) por reducir este problema, pero que a su vez contribuyen a mantener los prejuicios que existen en contra de las mujeres en roles de liderazgo. Esto sucede de manera inconsciente, razón por la cual es importante discutir este problema y permitir a líderes educativos y docentes cuestionar sus posibles prejuicios que los llevan a asumir que las mujeres generalmente se comportan de manera comunal cuando ocupan puestos de liderazgo, mientras los hombres se desempeñan mejor por ser más enérgicos al momento de ejercer el mando.

Mientras estas ideas continúen en las organizaciones y en las IEs será imposible incrementar la representación de la mujer en roles de liderazgo. Además, los problemas de inclusión, o la falta de inclusión, continuarán afectando a mujeres con estudios avanzados y experiencia profesional, así como a las organizaciones que necesitan un rango más amplio de talento para colocar personas en roles de liderazgo. Esto se ejemplifica en Pemberton et al. (2010), quienes analizaron las percepciones de estudiantes de posgrado en Estados Unidos respecto al grado de acoso y discriminación en sus instituciones educativas. Los autores concluyeron que tanto personal académico como administrativo discriminan a sus estudiantes de manera inconsciente debido a una falta de sensibilidad. Por ejemplo, estudiantes en la muestra del estudio citado comentaron que individuos con discapacidades preferían desertar debido a la falta de acceso apropiado a las instalaciones. Por otra parte, estudiantes de género femenino no enfrentaban problemas de acoso físico, pero tenían que soportar comentarios insensibles que las hacían sentir incomodas y entorpecían su relación con la institución. Esto no quiere decir que las estudiantes del estudio necesariamente fueran atacadas verbalmente. Según los autores, ellas notaban que algunos de sus profesores, inconscientemente, las trababan como personas inmaduras y con menos seriedad que a sus compañeros hombres.

Existe evidencia de diferencias en términos de rendimiento académico entre mujeres y hombres que se deben a factores socio-culturales que influyen sobre estos grupos (Coronado Ramírez, Sandoval Bravo \& Torres Mata, 2012). La literatura sugiere que este problema trasciende culturas. López Francés y Escalante Ferrer (2016) confirmaron la falta de equidad 
de género desde la perspectiva de estudiantes universitarios españoles y mexicanos. Las autoras descubrieron información sobre la persistencia de violencia de género entre estudiantes universitarios, así como tendencias por parte del personal académico de las instituciones educativas de tratar a los estudiantes de forma distinta de acuerdo a su género. La principal aportación del estudio, en términos de fijar la agenda de investigación, radica en la identificación del problema de falta de equidad de género que se sostiene y propaga por parte del personal académico de las universidades de manera sutil y no intencional.

Los estudios mencionados dan pie a suponer que existen sesgos dentro de las IEs que no se atienden de forma adecuada debido a que no se percibe una mala intención por parte de las personas involucradas. Estos sesgos fomentan comportamientos entre personal académico y administrativo que de una u otra forma reflejan estereotipos que afectan principalmente a las mujeres. Desde una perspectiva general, González Hurtado (2006) argumentó que el fomento de la inclusión en la educación en México requiere que los docentes favorezcan la diversidad sobre la igualdad y reconozcan la influencia de factores externos sobre el desempeño de los estudiantes. Esta idea refleja una actitud basada en el reconocimiento de que mujeres y hombres son diferentes, no iguales. Es precisamente el hecho de que son diferentes lo que agrega valor a los equipos gerenciales, lo que sugiere que es importante hacer un mayor esfuerzo por incrementar la representación e inclusión de profesionales de género femenino en roles de liderazgo.

En este sentido, es importante entender que es posible que los problemas que enfrentan las IES respecto a temas de equidad y justicia tienen que ver con problemas de inclusión y no necesariamente de discriminación (Sherbin \& Rashid, 2017). Es decir, aunque se han identificado avances importantes en términos de acceso a la educación, las experiencias educativas y el provecho que se obtiene de estas pueden ser diferentes para individuos que pertenecen a grupos demográficos o económicos subrepresentados. Tal es el caso de la preparación de la mujer que ingresa a la universidad con expectativas de egresar para ejercer puestos de liderazgo. Para fines de este estudio, el problema de falta de inclusión en la educación que se analiza es el que corresponde a la preparación de personas de género femenino para ejercer roles de liderazgo. La brecha de género en puestos directivos en México continúa siendo un reto por superar (Camarena Adame \& Saavedra García, 2018). Rincón, González y Barrero (2017) argumentaron que las trayectorias de mujeres se complican cuando terminan sus estudios y deciden emprender carreras profesionales 
en organizaciones donde existen barreras que parten de estereotipos que afectan el rol de la mujer. Este problema se puede abordar mediante estrategias educativas que preparen mejor a los individuos para ejercer roles de liderazgo en ambientes diversos e inclusivos y a través de la investigación educativa que examina los comportamientos de liderazgo de hombres y mujeres.

Vale la pena aclarar que en este manuscrito se reconoce la diferencia entre género y sexo. Ambos términos se utilizan a lo largo del documento para hacer referencia a personas de género masculino o femenino donde el término masculino se refiere a hombre, $\mathrm{y}$ femenino a mujer. Se reconoce la importancia de personas de la comunidad LGBTQ (Lesbian, Gay, Bisexual, Transexual, Queer) y es precisamente por esto que se deja su participación en puestos de liderazgo para otro estudio. En otras palabras, el término género se utiliza de la manera tradicional en este texto y se evita hacer referencia a otros como los de la comunidad LGBTQ, una de las limitaciones de este estudio.

Como punto de partida se toman las ideas de Eagly y Carli (2012), quienes han contribuido enormemente con la exploración del rol de la mujer en puestos de liderazgo. Estas autoras argumentaron que hoy en día es posible que las mujeres lleguen a ocupar puestos directivos en organizaciones de diferente tipo, pero su ascenso tiende a ser complicado y frustrante, como un laberinto (utilizando la metáfora de las autoras), situación que merma su desempeño en roles de liderazgo. En este estudio se busca documentar información que soporte que las mujeres y los hombres tienen competencias de liderazgo similares a pesar de los prejuicios frente a los estilos de liderazgo de mujeres. Con esto en mente, se aplicó una encuesta con 180 mujeres y 129 hombres estudiantes de posgrado en la ciudad de Tijuana, Baja California, México. Para recoger los datos, se utilizó el Inventario de Prácticas de Liderazgo desarrollado por Kouzes y Posner (2012). Los resultados representan autoevaluaciones de los estudiantes respecto a sus comportamientos de liderazgo, lo que permite formular supuestos que ayudan a líderes educativos y estudiantes a comprender mejor la importancia de este tipo de competencias y fomentar estrategias didácticas enfocadas en desarrollar habilidades directivas. Esto se logra a través del currículo y motivando la creación de una cultura institucional que valore la capacidad de liderazgo de los estudiantes, independientemente de su género. 


\section{La participación de las IES en el desarrollo de líderes}

Las IEs tienen como propósito preparar a sus estudiantes para tomar las riendas de los esfuerzos enfocados en abordar los problemas complejos que afectan a las organizaciones y a la sociedad en general (Andenoro, Sowcik \& Balser, 2017). Para cumplir este objetivo, es necesario priorizar el proceso educativo sobre los contenidos, promoviendo entre los estudiantes la capacidad para pensar de manera lógica y crítica, la búsqueda de alternativas y la adopción de enfoques sistémicos para la toma de decisiones que reflejen la realidad fuera del aula (Andenoro et al., 2017; Haber-Curran, Paige \& Tillapaugh, 2013). Complementando lo anterior, Fernández Batanero y Hernández Fernández (2013) describieron a los líderes inclusivos en centros educativos como individuos que promueven la diversidad, equidad, la mejora continua, el desarrollo profesional, la confianza y el trabajo en equipo.

Por su parte, Sherbin y Rashid (2017), en congruencia con la descripción del líder educativo de Ferrer, Clemenza, Romero y Rojas (2000), argumentaron que los líderes inclusivos se aseguran de que todos los integrantes del equipo se escuchan, toleran y aceptan propuestas novedosas, habilitan a sus seguidores para la toma de decisiones, escuchan y retroalimentan, y reconocen los logros. En este sentido, el personal docente, a través de diversas estrategias didácticas basadas en proyectos, es capaz de promover la inclusión y evaluarla (Gómez Hurtado \& Delgado Algarra, 2018). Sin embargo, en el caso de la preparación de mujeres que buscan ocupar puestos de liderazgo, es importante cuestionar aquello que tradicionalmente se ha asumido respecto a estilos de liderazgo, desempeño y género (Bass, 1981). En concreto, es importante que las culturas organizacionales de las IEs se dirijan en concordancia con los comportamientos típicos de líderes inclusivos, reduciendo los efectos negativos de los estereotipos y la discriminación. Esto se debe ver reflejado en la forma en que los docentes forman a sus estudiantes y los preparan para la vida y el trabajo.

El liderazgo en las IEs es un elemento esencial para la promoción de la diversidad y la inclusión (Ferrer et al., 2000; Fernández Batanero \& Hernández Fernández, 2013; Azorín Abellán, 2018). Las IEs tienen la responsabilidad de ayudar a formar personas capaces de desempeñar su trabajo de manera congruente con las necesidades de los diferentes tipos de organizaciones que operan en su entorno. Para tomar ventaja del potencial de 
las personas que forman una organización, es importante promover la diversidad y la inclusión. Esto implica contar con equipos de trabajo compuestos por individuos con características y experiencias diferentes, lo que lleva a promover la diversidad de género, edad, lugar de origen, ideología, y el fomento de prácticas directivas inclusivas que lleven a la participación activa de todos para potencializar sus diferencias (Sherbin \& Rashid, 2017). En este sentido, es de suponer que las IEs buscan aprovechar, en lugar de suprimir o ignorar, las diferencias entre sus estudiantes.

Los programas educativos diseñados para el fomento del liderazgo deben partir de un diagnóstico que permita identificar similitudes y diferencias entre las personas que se busca atender. Seemiller y Murray (2013) recomendaron el uso de cuestionarios de competencias de liderazgo en versión de auto-reporte para diagnosticar las necesidades de estudiantes en este rubro. Los autores argumentaron que los coordinadores de programas educativos pueden utilizar los resultados de diferentes grupos de estudiantes como indicadores de autoeficacia de liderazgo que sirvan para hacer comparaciones y diseñar intervenciones educativas de forma congruente.

\section{La formación de mujeres en roles de líder}

La Organización para la Cooperación y Desarrollo Económico — OCDE- (2017) documentó el progreso de las mujeres en el entorno político en México. Según esta organización, en un periodo de 10 años, la participación de mujeres en la Cámara de Representantes aumentó de $22.6 \%$ a $42 \%$, mientras que el aumento en el Senado fue de $17.2 \%$ a $33.6 \%$. Sin embargo, la misma fuente advierte que este incremento se debe principalmente a la implementación de cuotas de género y no necesariamente a métricas de desempeño o cambio de valores. Esto sugiere que existe un reconocimiento de los problemas que implica la histórica subrepresentación de la mujer en el entorno político del país y que la sociedad se ha tomado en serio la iniciativa para disminuir la brecha entre hombres y mujeres, pero no habla de prácticas de inclusión diseñadas para potencializar la contribución de las mujeres en roles de liderazgo. Sin embargo, las señales son favorables, especialmente el hecho que, según el Foro Económico Mundial — FEM- (2018), la paridad entre mujeres y hombres en el rubro de educación es prácticamente perfecta. 
Sin embargo, mientras los retos de representación o participación comienzan a ser superados, los problemas de inclusión y éxito continúan en la educación y el trabajo. Existe un alto índice de deserción en la transición de educación media a educación superior en mujeres y continúan subrepresentadas en el mercado laboral y educativo en disciplinas de ciencia, ingeniería, tecnología y matemáticas (ocDE, 2017). El FEM (2018) utiliza una escala que va de 0 (menor paridad) a 1 (paridad total) para medir la brecha de género por país para las dimensiones: participación económica y oportunidad, educación, salud y participación política. En este indicador, México recibió una calificación general en 2018 de .721, por debajo de Cuba (.749), Bolivia (.748), Costa Rica (.749), y Colombia y Ecuador (ambos con .729).

Bass (1981) puntualizó los logros de la mujer en educación y negocios, pero advirtió que el hecho de que los puestos directivos en organizaciones se encuentren cada vez más balanceados en términos de género no significa que las aportaciones de las mujeres directivas tengan el mismo nivel de aceptación o impacto para la toma de decisiones que las contribuciones de sus colegas de género masculino. Posteriormente, Pratch y Jacobowitz (1996) concluyeron que las mujeres en puestos de liderazgo tenían que ajustar sus comportamientos en mayor medida que los hombres debido a los estereotipos que perduran a pesar del gran avance en términos de igualdad en las últimas décadas. Existe una línea de investigación enfocada en el cambio evolutivo de la sociedad que lleva a un entorno más equitativo entre mujeres y hombres (Fetterolf \& Eagly, 2011; Eagly \& Wood, 2013; 2017). Algunos de los estudios en esta línea se enfocan en cuestiones de liderazgo de la mujer. Por ejemplo, Cárdenas de Santamaría, Eagly, Heller, Salgado, Jáuregui y Goode (2013) entrevistaron a 162 mujeres directivas en 17 países de América Latina para identificar los factores que las llevaron a escalar a puestos de liderazgo. Las autoras concluyeron que las líderes de su muestra lograron avanzar en sus carreras mediante la realización de estudios de posgrado, el balance efectivo de vida y carrera, y el desarrollo de una visión de líder acompañado de una orientación en resultados.

Anteriormente se han realizado estudios sobre género y liderazgo enmarcados en la teoría de liderazgo transformacional (Eagly \& Carli, 2012; Eagly \& Johannesen-Schmidt, 2001; Cuadrado \& Molero, 2002; Díaz, 2018). Asimismo, las conductas de líderes transformacionales han emergido en estudios exploratorios sobre liderazgo en contextos educativos (Gómez, 2019). Eagly y Johannesen-Schmidt (2001) argumentaron que las mujeres 
tienden a adoptar comportamientos de liderazgo más transformacionales que los hombres, situación que sugiere que parte de la razón por la que las mujeres experimentan mayor nivel de rechazo en las organizaciones se debe a su estilo de liderazgo. Sin embargo, las autoras advirtieron que las diferencias en términos de liderazgo transformacional, por género, son mínimas; subsecuentes investigaciones concluyeron que no hay diferencias significativas entre hombres y mujeres con base en modelos transformacionales (García Solarte, Salas-Arbeláez \& Gaviria Martínez, 2017; Díaz, 2018).

\section{Liderazgo transformacional}

Bass (1981) desarrolló un análisis extenso de los principales enfoques teóricos de liderazgo. Su trabajo incluye los modelos de personalidad, comportamiento, situacional y transformacional. A pesar de que existen varios modelos de liderazgo que difieren en aspectos importantes (por ejemplo, si el liderazgo es una característica con la que se nace o se desarrolla mediante la experiencia y la educación), es claro que los líderes facilitan el movimiento de las masas que llevan al cambio social (Burns, 2010). En el caso de la subrepresentación y falta de inclusión de la mujer en roles de liderazgo, Bass (1981) desmintió que se deba a que el género sea una determinante de efectividad o interés. Es decir, el hecho de ser mujer no implica una desventaja con respecto al desempeño efectivo en puestos de liderazgo y tampoco implica que las mujeres, por el hecho de ser mujeres, evitan participar en roles directivos.

Entre los enfoques teóricos analizados por Bass (1981) destaca el modelo transformacional por su énfasis en valores, el rol de los seguidores, desempeño y equidad. Burns (2010) es considerado uno de los principales exponentes de la teoría de liderazgo transformacional y fue él quien popularizó la teoría a finales de la década de 1970. Para él, el liderazgo efectivo implica la participación del líder y sus seguidores, y fue claro cuando afirmó que los grandes cambios son llevados a cabo por las masas y no por individuos trabajando de forma unilateral. Estas concepciones de liderazgo, el líder y el seguidor han ido cobrando fuerza con el tiempo. Por su parte, Cruz Ortiz, Salanova y Martínez (2013) recomendaron la incorporación de la teoría de liderazgo transformacional en investigaciones de clima organizacional y satisfacción. Kellerman (2012) advirtió que los líderes efectivos hoy en día han reducido su nivel de influencia sobre sus seguidores. La idea central de la autora es que el rol del líder moderno es administrar, integrar y alentar las 
iniciativas de las personas que se encuentran a su cargo y fomentar el desarrollo de nuevos líderes. Por su parte, Heifetz (1994) advirtió que los sistemas de autoridad tradicionales, donde se asume que las personas en puestos de autoridad tienen mayor capacidad para abordar los problemas que enfrentan las organizaciones, pueden ser contraproducentes.

Para Heifetz (1994), la complejidad en los contextos de negocios, políticos y educativos requieren de un enfoque sistémico donde los seguidores aprovechen su capacidad de diagnóstico y adaptación para encontrar soluciones que no siempre están al descubierto. Nelson y Squires (2017) argumentaron que el modelo de liderazgo adaptativo originalmente propuesto por Heifetz es apropiado en el contexto educativo por su enfoque en la solución de problemas, relaciones dinámicas entre líderes y seguidores, y su congruencia con el entorno educativo actual. Por tanto, a partir de la década de 1970, se acepta que el liderazgo es una competencia que se basa en comportamientos que pueden ser desarrollados mediante la experiencia, la educación y el apoyo de mentores (Bass, 1981). Asimismo, el liderazgo se define desde una óptica más amplia donde el líder no es necesariamente la persona en un puesto de autoridad, sino la persona que influye sobre los demás para promover cambios deseables en organizaciones y en la sociedad en general (Burns, 2010). Con esto en mente, Kouzes y Posner (2012) dedicaron parte de sus carreras a identificar los comportamientos que llevaron a líderes de diferentes industrias a lograr altos niveles de desempeño. Como resultado de sus investigaciones, desarrollaron su propio modelo de liderazgo transformacional: Cinco Prácticas de Liderazgo Ejemplar.

El modelo de Kouzes y Posner (2012) incluye cinco prácticas que los lideres efectivos implementan mediante su comportamiento: (1) modelar el camino, (2) inspirar una visión compartida, (3) retar el proceso, (4) habilitar a los demás para que actúen y (5) alentar el corazón. Dado que se trata de un modelo de liderazgo transformacional, la intención principal del líder que se comporta de manera congruente con las cinco prácticas es la transformación positiva y personalizada de sus seguidores. En congruencia con Burns (2010), tanto los líderes transformacionales como sus seguidores se elevan unos a otros a niveles superiores de desempeño como resultado de su interacción. Como se observa en la tabla 1, el modelo de las cinco prácticas de liderazgo ejemplar tiene importantes coincidencias con la propuesta de líderes inclusivos que ofrece Catalyst (2015). 
Tabla 1. Congruencia entre el líder transformacional y el líder inclusivo

\begin{tabular}{lll}
\hline Práctica de liderazgo & Kouzes y Posner (2012) & Catalyst (2015) \\
\hline Modelar el camino & El líder actúa con base en principios. & $\begin{array}{l}\text { El líder inclusivo promueve que se haga lo } \\
\text { correcto. }\end{array}$ \\
\hline $\begin{array}{l}\text { Inspirar una visión } \\
\text { compartida }\end{array}$ & $\begin{array}{l}\text { El líder genera consenso entre inte- } \\
\text { grantes del equipo y lo articula en el } \\
\text { largo plazo. }\end{array}$ & $\begin{array}{l}\text { El líder inclusivo promueve las aportaciones } \\
\text { de los demás, valorando ideas nuevas y difer- } \\
\text { entes. }\end{array}$ \\
\hline Retar el proceso & $\begin{array}{l}\text { El líder busca alternativas y acepta } \\
\text { retos que valen la pena. }\end{array}$ & $\begin{array}{l}\text { El líder inclusivo toma riesgos calculados y } \\
\text { promueve la diversidad. }\end{array}$ \\
\hline $\begin{array}{l}\text { Habilitar a los demás } \\
\text { para que actúen }\end{array}$ & $\begin{array}{l}\text { El líder empodera a los integrantes } \\
\text { del equipo para tomar decisiones. }\end{array}$ & $\begin{array}{l}\text { El líder inclusivo asigna tareas y da confianza a } \\
\text { sus seguidores, a quienes considera capaces } \\
\text { de lograr sus metas. }\end{array}$ \\
\hline Alentar el corazón & El líder celebra los logros de los & $\begin{array}{l}\text { El líder inclusivo fomenta un sentido de perte- } \\
\text { necia a través de la organización. }\end{array}$ \\
\hline
\end{tabular}

\section{Hipótesis}

Con base en el propósito del estudio y el modelo de las cinco prácticas de liderazgo ejemplar, se plantearon las siguientes hipótesis:

H1: Existen diferencias estadísticamente significativas entre mujeres y hombres de la muestra con respecto a la práctica de liderazgo modelar el camino.

H2: Existen diferencias estadísticamente significativas entre mujeres y hombres de la muestra con respecto a la práctica de liderazgo inspirar una visión compartida.

H3: Existen diferencias estadísticamente significativas entre mujeres y hombres de la muestra con respecto a la práctica de liderazgo retar el proceso.

H4: Existen diferencias estadísticamente significativas entre mujeres y hombres de la muestra con respecto a la práctica de liderazgo habilitar a los demás para que actúen.

H5: Existen diferencias estadísticamente significativas entre mujeres y hombres de la muestra con respecto a la práctica de liderazgo alentar el corazón. 


\section{Método}

Este estudio sigue una metodología cuantitativa no probabilística mediante la aplicación de una encuesta diseñada para medir la frecuencia con la que los participantes se comportan de manera congruente con un modelo transformacional de liderazgo. El propósito fue evaluar las diferencias entre mujeres y hombres de la muestra en términos de liderazgo transformacional, particularmente con base en el modelo de las cinco prácticas de liderazgo ejemplar de Kouzes y Posner (2012). Esta metodología es apropiada cuando se busca analizar datos de una muestra específica sin intención de generalizar los resultados en poblaciones más amplias; sin embargo, los resultados pueden ser útiles para acotar brechas de conocimiento específicas de interés para ciertos públicos (Creswell, 2014).

La muestra seleccionada para este estudio se compone de 180 mujeres y 129 hombres, todos estudiantes de posgrado en la ciudad de Tijuana, Baja California, México. De los 309 participantes, 254 estudiaban la maestría en administración, mientras que 55 estudiaban la maestría en educación. Se seleccionó esta muestra por su estatus como estudiantes de posgrado. Normalmente, estos individuos se preparan para ocupar puestos gerenciales en el corto o mediano plazo, y una expectativa de sus programas es precisamente desarrollar las habilidades directivas necesarias para desemperñarse de manera efectiva en dichos cargos.

El acopio de datos se llevó a cabo en los salones de clase de los participantes, con el apoyo de sus coordinadores de programa académico y personal docente. Antes de que los estudiantes aceptaran participar en el estudio, se les entregó un formulario de consentimiento informado donde se explicaba por escrito el propósito de la investigación y la promesa de confidencialidad. Posteriormente, los participantes recibieron instrucciones detalladas para llenar el cuestionario. El acopio de datos se llevó a cabo utilizando la versión impresa del IPL en versión auto-reporte.

El IPL se compone de 30 reactivos que se evalúan con una escala de Likert que va del 1 (casi nunca) hasta el 10 (casi siempre) para determinar la frecuencia con la que los participantes practican los comportamientos descritos por los reactivos. Cada reactivo corresponde a un comportamiento de liderazgo transformacional, que forman cinco constructos que corresponden al modelo de Kouzes y Posner (2012), es decir, cada constructo 
se compone de seis reactivos. El rango de puntos que puede obtener cada participante por constructo es de 0 a 60 . El IPL fue utilizado en este estudio con el permiso por escrito de la editorial y de los autores. Este ha sido ampliamente utilizado en contextos educativos (Wisner, 2011; Quin et al., 2015; McKinney \& Waite, 2016; Romsa et al., 2017), lo que habla de su validez. Wisner (2011) examinó las competencias de líderes estudiantes mediante el IPL en su versión de auto-reporte. La autora concluyó su análisis haciendo notar que la autoevaluación permite a los estudiantes establecer objetivos que les facilitan el desarrollo en sus habilidades de liderazgo.

Para realizar el análisis de datos, primero se examinó la fiabilidad de los cinco constructos del IPL con el propósito de asegurar que tuvieran niveles aceptables de consistencia interna. Posteriormente, se calcularon promedio y desviación estándar por constructo para determinar el nivel de frecuencia para cada comportamiento del liderazgo. Finalmente, para identificar diferencias entre los participantes de género femenino y masculino, se corrieron pruebas $t$ de muestras independientes por constructo. La prueba $t$ de muestras independientes es comúnmente utilizada para la comprobación de hipótesis que requiere comparar promedios en escalas de dos grupos (Jaeger, 1983). El proceso fue asistido mediante el uso del software spss, versión 23.

\section{Resultados}

El total de cuestionarios completados fue 309, lo que representa aproximadamente el $30 \%$ de la población de estudiantes de posgrado en las IEs involucradas. En la tabla 2 se presenta estadística descriptiva y coeficiente de Cronbach. Como se puede observar, cuatro de los cinco constructos cuentan con índices de fiabilidad por encima del estándar $(\alpha \geq 70)$, pero uno de los constructos, habilitar a los demás para que actúen, tuvo un índice por debajo del mínimo aceptable $(\alpha<.70)$. 
Tabla 2. Promedio, desviación estándar, y alfa de Cronbach del IPL

\begin{tabular}{lcccc}
\multicolumn{1}{c}{ Prácticas de liderazgo } & N & M & DE & $\boldsymbol{\alpha}$ \\
\hline Modelar el camino & 309 & 44.79 & 7.83 & .73 \\
\hline Inspirar una visión compartida & 309 & 41.88 & 9.66 & .80 \\
\hline Retar el proceso & 309 & 43.87 & 8.65 & .75 \\
\hline Habilitar a los demás para que actúen & 309 & 50.25 & 5.79 & .65 \\
\hline Alentar el corazón & 309 & 48.09 & 8.47 & .84 \\
\hline
\end{tabular}

Los resultados de la prueba $t$ de muestras independientes (tabla 3) permiten retener $\mathrm{H} 1$ y $\mathrm{H} 5$, aceptando un margen de error del 10\% ( $\mathrm{p} \leq .10)$, mientras que se rechazan $\mathrm{H} 2$, H3 y H4 (p > .10).

Tabla 3. Prueba t de muestras independientes de mujeres y hombres que completaron el IPL

\begin{tabular}{lccccccc}
\hline & \multicolumn{3}{c}{ Femenino } & \multicolumn{4}{c}{ Masculino } \\
\hline Prácticas de liderazgo & $\mathrm{N}$ & $\mathrm{M}$ & $\mathrm{DE}$ & $\mathrm{N}$ & $\mathrm{M}$ & $\mathrm{DE}$ & $\mathrm{t}$ \\
\hline Modelar el camino & 180 & 45.42 & 7.80 & 129 & 43.91 & 7.83 & $1.67^{\star}$ \\
\hline Inspirar una visión compartida & 180 & 42.08 & 9.89 & 129 & 41.59 & 9.36 & .44 \\
\hline Retar el proceso & 180 & 43.93 & 9.28 & 129 & 43.78 & 7.73 & .16 \\
\hline Habilitar a los demás para que actúen & 180 & 50.13 & 6.20 & 129 & 50.41 & 5.18 & -.43 \\
\hline Alentar el corazón & 180 & 48.88 & 8.91 & 129 & 47.00 & 7.71 & $1.93^{\star}$ \\
\hline
\end{tabular}

Nota: *estadísticamente significativo $(p<.10)$.

\subsection{Implicaciones para líderes educativos y estudiantes}

Los resultados sugieren que los comportamientos de mujeres y hombres en la muestra son similares. Las mujeres obtuvieron promedios superiores y estadísticamente significativos $(\mathrm{p}<.10)$ en dos de los cinco constructos que corresponden a las prácticas de modelar el camino y alentar el corazón. La principal conclusión que se obtiene del resultado es que mujeres y hombres tienen percepciones similares respecto a sus conductas de liderazgo, por tanto, se asume que son capaces de desarrollar este tipo de competencia como parte de la experiencia o estudios universitarios para desempeñarse de manera efectiva en roles de liderazgo. Esto es congruente con los trabajos de Eagly y Carli (2012), Eagly y Johannesen-Schmidt (2001), Cuadrado y Molero (2002), y Díaz (2018). 
Manfredi (2017) argumentó que el problema de baja participación de mujeres en puestos directivos se puede abordar mediante un proceso reflexivo y complejo que termina en una contextualización amplia e inclusiva de lo que se considera liderazgo exitoso y éxito organizacional. Este proceso se facilita cuando se descartan ideas basadas en estereotipos que distorsionan la realidad. Este estudio y otros citados anteriormente contribuyen a contrarrestar el peso de los estereotipos y prejuicios que obstaculizan la reflexión objetiva respecto a la participación de mujeres en roles de liderazgo y las implicaciones para IEs. Esto no es únicamente un ejercicio de justicia social, sino un argumento que favorece el desempeño de los equipos directivos en organizaciones que se benefician de la diversidad de sus integrantes.

Por ejemplo, Ruiz-Jiménez, Fuentes-Fuentes y Ruiz-Arroyo (2016) encuestaron a 205 representantes de organizaciones de la industria de telecomunicaciones en España para determinar cómo impacta la diversidad de género en los equipos directivos de este tipo de organización. Las autoras concluyeron que los equipos directivos se benefician de la diversidad de género, situación que se manifiesta en términos de desempeño, innovación y creatividad. Con esto en mente y aprovechando los resultados del presente estudio, así como las contribuciones previamente citadas, es posible proponer lo siguiente a estudiantes y personal que trabaja en IES para contribuir a acotar la brecha de género en puestos directivos.

1. Promover el liderazgo transformacional. Los resultados de este estudio sugieren que tanto mujeres como hombres son similares en términos de su apreciación respecto a conductas transformacionales de liderazgo. En este sentido, comportamientos enfocados en modelar el camino, inspirar una visión compartida, retar el proceso, habilitar a los demás para que actúen y alentar el corazón son comportamientos que se deben fomentar mediante tareas en equipo y proyectos donde los docentes enfaticen la importancia del rol del líder en el desempeño de grupo.

2. Estudios de posgrado. Las mujeres que desean ocupar puestos de liderazgo en algún momento de sus carreras deben considerar estudiar un posgrado como parte de la estrategia de contrarrestar los prejuicios que continúan obstaculizando el rol de la mujer directiva. Moncayo Orjuela y Zuluaga Goyeneche (2015) analizaron los perfiles directivos de 90 líderes educativas de siete IES en Colombia y concluyeron que estudiar un posgrado representa una forma de rebeldía por parte de la mujer que busca romper 
barreras de género que tradicionalmente favorecen al sexo masculino en puestos directivos. Al observar que los puestos de alta dirección son ocupados principalmente por hombres, las mujeres contrarrestan este desbalance parcialmente mediante sus estudios.

3. Alinear intereses personales y familiares. Montes-de-Oca-O'Reilly y Yurén Camarena (2010) examinaron las trayectorias de 12 líderezas educativas para generar recomendaciones para el fomento de desarrollo de competencias de liderazgo entre mujeres. A manera de resumen, las recomendaciones presentadas por las autoras son fomentar experiencias conducentes al desarrollo de liderazgo en la familia, alinear las actividades en contextos educativos con el fomento al liderazgo de la mujer y participar en procesos formativos fuera de casa y de la escuela.

4. Capacitar al personal docente y administrativo en IEs. Cuevas-López y DíazRosas (2015) afirmaron que, si bien es cierto que las mujeres y hombres tienen diferentes estilos de liderazgo, esto no implica que el desempeño del líder depende de su género. Asimismo, reconocieron el trabajo que se lleva a cabo en la Universidad Complutense de Madrid por promover la equidad de género y argumentaron que incrementar la participación de la mujer en puestos de liderazgo es un tema que debe ocupar a las IEs. Estos esfuerzos por parte del personal que trabaja en ies deben ser replicados para asegurar que todos los estudiantes se sientan valorados con respecto a su potencial para desarrollar competencias de liderazgo.

5. Difundir estudios empíricos sobre liderazgo y género. Sánchez Moreno y López Yáñez (2008) analizaron los comportamientos de 136 mujeres directivas en instituciones educativas. Sus principales hallazgos fueron que sus participantes destacan por su capacidad para explotar sus relaciones interpersonales, hacer valer su autoridad y realizar múltiples actividades a la vez. En otro estudio, Díez Gutiérrez, Terrón Bañuelos y Anguita Martínez (2009) también apoyaron el argumento a favor de la tendencia de mujeres directivas que enfocan su estilo de liderazgo hacia conductas dirigidas a reforzar relaciones interpersonales. Estos estudios, acompañados del trabajo de Eagly y Carli (2012) son buenos ejemplos encontrados en la literatura que dan soporte empírico a la noción que tanto mujeres como hombres son capaces de ejercer roles de liderazgo y ayudan a desmentir ideas 
equivocadas basadas en estereotipos que históricamente han creado obstáculos para mujeres que aspiran y ocupan puestos directivos.

\section{Conclusión}

En este estudio se abordó el problema de la falta de inclusión de la mujer en roles de liderazgo. A pesar de que las mujeres incrementan su participación en equipos directivos y como estudiantes en IEs, todavía existen estereotipos que se manifiestan de diferentes maneras en organizaciones e incluso dentro de las IEs. Esto obstaculiza el progreso en términos de equidad de género en general, específicamente en mujeres que aspiran a ejercer roles de liderazgo. Para disminuir el impacto de los estereotipos y potencializar la diversidad de talento en equipos directivos se recomienda implementar estrategias educativas encaminadas al desarrollo de competencias de liderazgo transformacional. El enfoque en modelos de liderazgo transformacional se recomienda por tratarse de prácticas o comportamientos universales que, según varios estudios empíricos citados en este estudio, son congruentes con los géneros femenino y masculino por lo que se logra minimizar el impacto de prejuicios en contra de algún grupo.

Con base en los resultados de IPL con la muestra de 309 estudiantes de posgrado que participaron en este estudio, se sugiere que la percepción de mujeres y hombres respecto a sus conductas de liderazgo transformacional son simulares, lo que a su vez sugiere que el género no debe ser una limitante para fomentar el desarrollo de competencias de liderazgo entre estudiantes. Este resultado se agrega al trabajo de varios autores que trabajan con base en la teoría de liderazgo transformacional que sostiene que el género no influye en la autovaloración de comportamientos de liderazgo.

Entre las limitantes que se identifican en este trabajo se incluyen las siguientes: primero, el constructo habilitar a los demás para que actúen tuvo un índice de consistencia interna relativamente bajo $(\alpha<.70)$ lo que sugiere que los resultados correspondientes deben someterse a escrutinio. Segundo, al tratarse de una muestra de conveniencia, los resultados solamente aplican a la población bajo estudio, no pueden generalizarse. Tercero, los niveles 
de confianza se establecieron en $90 \%$ (margen de error $\leq .10$ ) lo cual es aceptable en algunos casos, pero es recomendable analizar los datos con niveles de $95 \%$ para dar mayor certeza a los resultados. Estas limitantes se pueden abordar en investigaciones futuras.

\section{Referencias}

Andenoro, A. C., Sowcik, M. J., \& Balser, T. C. (2017). Addressing complex problems: Using authentic audiences and challenges to develop adaptive leadership and socially responsible agency in leadership learners. Journal of Leadership Education, 16(4), 1-19.

Azorín Abellán, C. M. (2018). Percepciones docentes sobre la atención a la diversidad: Propuestas desde la práctica para la mejora de la inclusión educativa. Ensayos: Revista de la Facultad de Educación de Albacete, 33(1), 173-188.

Bass, B. M. (1981). Stogdill's handbook of leadership: A survey of theory and research (revised and expanded edition). New York: Free Press.

Burns, J. (2010). Leadership. New York: Harper Perennial Political Classics.

Camarena Adame, M. E., \& Saavedra García, M. L. (2018). El techo de cristal en México. Revista de Estudios de Género. La Ventana, 5(47), 312-347.

Cárdenas de Santamaría, M. C., Eagly, A. H., Heller, L., Salgado, E., Jáuregui, K., \& Goode, W. (2013). Claves para el ascenso de las altas ejecutivas en América Latina. INCAE Business Review, 2(9), 52-56.

Catalyst. (2015). Inclusion matters. Recuperado de https://www.catalyst.org/system/files/ inclusion_matter.pdf

Coronado Ramírez, S., Sandoval Bravo, S., \& Torres Mata, A. (2012). Diferencias de género, factores que inciden en el rendimiento matemático de licenciaturas económico administrativas. Sinéctica, (39), 1-24.

Creswell, J. W. (2014). Research design: Qualitative, quantitative, and mixed methods approaches. Thousand Oakes: Sage Publications.

Cruz Ortiz, V., Salanova, M., \& Martínez, I. M. (2013). Liderazgo transformacional: Investigacion actual y retos futuros. Universidad \& Empresa, 25, 13-32.

Cuadrado, I., \& Molero, F. (2002). Liderazgo transformacional y género: Autoevaluaciones de directivos y directivas españoles. Revista de Psicología del Trabajo y de las Organizaciones, 18(1), 39-55. 
Cuevas-López, M., \& Díaz-Rosas, F. (2015). Género y liderazgo en la universidad española. Un estudio sobre la brecha de género en la gestión universitaria. Education Policy Analysis Archives, 1-22.

Díaz, Eduardo R. (2018). Leadership self-efficacy: A study of male and female mBA students in Mexico. Advancing Women in Leadership, 38(9), 27-34.

Díez Gutiérrez, E. J., Terrón Bañuelos, E., \& Anguita Martínez, R. (2009). Percepción de las mujeres sobre el "techo de cristal" en educación. Revista Interuniversitaria de Formación del Profesorado, 23(1), 27-40.

Eagly, A. H., \& Carli, L. L. (2012). Women and the labyrinth of leadership. Contemporary Issues in Leadership, 147-162.

Eagly, A. H., \& Johannesen-Schmidt, M. C. (2001). The leadership styles of women and men. Journal of Social Issues, 57(4), 781.

Eagly, A. H., \& Wood, W. (2013). Feminism and evolutionary psychology: Moving forward. Sex Roles, 69(9-10), 549-556.

Eagly, A. H., \& Wood, W. (2017). Janet Taylor Spence: Innovator in the study of gender. Sex Roles, 77(11-12), 725-733.

Fernández Batanero, J. M., \& Hernández Fernández, A. (2013). El liderazgo como criterio de calidad en la educación inclusiva. Estudios Sobre Educación, (24), 83-102.

Ferrer, J., Clemenza, C., Romero, D., \& Rojas, L. R. (2000). Liderazgo corporativo y crisis paradigmática universitaria. Convergencia Revista de Ciencias Sociales, (23).

Fetterolf, J. C., \& Eagly, A. H. (2011). Do young women expect gender equality in their future lives? An answer from a possible selves experiment. Sex Roles, 65(1-2), 83-93.

García Solarte, M., Salas-Arbeláez, L., \& Gaviria Martínez, E. (2017). Estilos de liderazgo de hombres y mujeres en las pymes. Ad-Minister, (31), 25-46.

Gómez, L. F. (2019). Trenzar los hilos de la escuela. El liderazgo, elemento clave para el funcionamiento de los consejos técnicos escolares. Sinéctica, (52), 1-20.

Gómez Hurtado, I., \& Delgado Algarra, E. J. (2018). Atender a la diversidad desde la enseñanza de las ciencias sociales: Un enfoque inclusivo. Ensayos: Revista de la Facultad de Educación de Albacete, 33(1), 103-111.

González Hurtado, R. (2006). ¿Pueden nuestras escuelas promover la inclusión? Sinéctica, (29), 28-35.

Haber-Curran, P., \& Tillapaugh, D. (2013). Leadership learning through student-centered and inquiry-focused approaches to teaching adaptive leadership. Journal of Leadership Education, 12(1), 92-116. 
Heifetz, R. A. (1994). Leadership without easy answers. Cambridge: Harvard University Press.

Jaeger, R. M. (1983). Statistics: A spectator sport. Beverly Hills: Sage Publications.

Kellerman, B. (2012). The end of leadership. New York: HarperCollins Publishers.

Kouzes, J. M., \& Posner, B. Z. (2012). The leadership challenge: How extraordinary things bappen in organizations. San Francisco: Leadership Challenge.

López Francés, I., \& Escalante Ferrer, A. E. (2016). Análisis comparativo entre universidades Española y Mexicana. Sinéctica, (46), 1-20.

Manfredi, S. (2017). Increasing gender diversity in senior roles in HE: Who is afraid of positive action? Administrative Sciences, 7(2), 19.

McKinney, N. S., \& Waite, R. (2016). Leadership development among a cohort of undergraduate interdisciplinary students in the health professions. Journal of Leadership Education, 15(3), 11-22.

Moncayo Orjuela, B. C., \& Zuluaga Goyeneche, D. (2015). Estilos de liderazgo en cargos universitarios, estudio descriptivo con mujeres directivas. Revista Panorama, 9(17), 74-84.

Montes-de-Oca-O'Reilly, A., \& Yurén Camarena, T. (2010). Trayectancia y formación de liderazgo en académicas mexicanas: El caso Morelos. Education Policy Analysis Archives, 18(12/13), 1-31.

Nelson, T., \& Squires, V. (2017). Addressing complex challenges through adaptive leadership: A promising approach to collaborative problem solving. Journal of Leadership Education, 16(4), 111-123.

OECD. (2017). The status of women in Mexico. En Building an inclusive Mexico: Policies and good governance for gender equality (pp. 53-86). Paris: OECD Publishing.

Pemberton, C., Ray, B., Said, H., Easterly, D., \& Belcher, C. (2010). Graduate student perceptions of college of education culture and climate. Advancing Women in Leadership, 30(5), 1-23.

Pratch, L., \& Jacobowitz, J. (1996). Gender, motivation, and coping in the evaluation of leadership effectiveness. Consulting PsychologyJournal: Practice and Research, 48(4), 203-220.

Quin, J., Deris, A., Bischoff, G., \& Johnson, J. T. (2015). Comparison of transformational leadership practices: Implications for school districts and principal preparation programs. Journal of Leadership Education, 14(3), 71-85.

Rincón, V., González, M., \& Barrero, K. (2017). Women and leadership: Gender barriers to senior management positions. Intangible Capital, 13(2), 319-386. 
Romsa, B., Romsa, K., Lim, J., \& Wurdinger, S. (2017). Undergraduate sport management students' perceptions of leadership skills through service learning. Journal of Leadership Education, 16(2), 129-147.

Ruiz-Jiménez, J. M., Fuentes-Fuentes, M. M., \& Ruiz-Arroyo, M. (2016). Knowledge combination capability and innovation: The effects of gender diversity on top management teams in technology-based firms. Journal of Business Ethics, 135(3), 503-515.

Sánchez Moreno, M. S., \& López Yáñez, J. (2008). Poder y liderazgo de mujeres responsables de Instituciones Universitarias. Revista Española de Pedagogía, (240), 345-364.

Seemiller, C., \& Murray, T. (2013). The common language of leadership. Journal of Leadership Studies, 7(1), 33-45.

Sherbin, L., \& Rashid, R. (2017). Diversity doesn't stick without inclusion. Harvard Business Review Digital Articles, 2-5.

Wisner, M. D. (2011). Psychological strengths as predictors of effective student leadership. Christian Higher Education, 10(3/4), 353-375.

WEF. (2018). The global gender gap report 2018. Geneva: Autor. 